\title{
Timing of surgery in infective endocarditis with cerebral complications: Time to think outside the nonexistent box
}

\author{
Maroun Yammine, MD, Tsuyoshi Kaneko, MD, and Sary Aranki, MD
}

\author{
From the Division of Cardiac Surgery, Brigham and Women's Hospital, Boston, Mass. \\ Disclosures: Authors have nothing to disclose with regard to commercial support. \\ Received for publication July 24, 2017; accepted for publication July 31, 2017; available ahead of print Sept 8, \\ 2017. \\ Address for reprints: Sary Aranki, MD, Division of Cardiac Surgery, Brigham and Women's Hospital, 75 Francis \\ St, Boston, MA 02115 (E-mail: saranki@ partners.org). \\ J Thorac Cardiovasc Surg 2018;156:601 \\ $0022-5223 / \$ 36.00$ \\ Copyright (C) 2017 Published by Elsevier Inc. on behalf of The American Association for Thoracic Surgery \\ http://dx.doi.org/10.1016/j.jtcvs.2017.07.061
}

In the April 2017 issue of the Journal, Murai and coworkers $^{1}$ report on the effect of the interaction between stroke severity and timing of surgery on clinical outcomes in patients with active infective endocarditis (IE) and cerebral complications with moderate neurologic deficits. Their retrospective study cohort consisted of 170 consecutive patients from a single institution during a 24-year period starting in 1990. The National Institutes of Health Stroke Scale was used to evaluate the severity of stroke. The modified Rankin scale was used to determine residual disability at discharge. Most of the patients $(80 \%)$ had moderate stroke. Almost half of those patients underwent early surgery for IE, defined as within 2 weeks of onset. The remainder had the IE conventionally managed. Early and late clinical outcomes were significantly better for the early surgical group. Murai and coworkers ${ }^{1}$ conclude that early surgery could be beneficial for patients with moderate strokes.

Secondary neurologic exacerbation related to systemic anticoagulation and hypotension associated with cardiopulmonary bypass are the main concerns for avoiding surgery after a stroke with IE. Widening of the cerebral infarct or intracerebral bleed areas and possible hemorrhagic conversions of an infarcted area are valid concerns, yet they have not been substantiated by any well-controlled studies. By using the National Institutes of Health Stroke Scale, Murai and coworkers ${ }^{1}$ have introduced a novel tool to allow an objective method of documenting stroke severity and assessing the impact of early IE surgery on clinical outcomes. We all agree that the results associated with severe stroke are dismal, regardless of the management strategy. It appears that most strokes are moderate $(80 \%)$, however, and almost half are asymptomatic. Neurologic outcomes in patients undergoing early surgery, were excellent regardless of whether the patients had symptoms, or whether a cerebral infarct or hemorrhage was the responsible pathologic entity.

Another strength of this study is that it highlights the extent of asymptomatic septic embolization. The implications are enormous for diagnosis and timing of IE surgery. Routine cerebral and systemic imaging should become part and parcel of the management algorithm of patients with

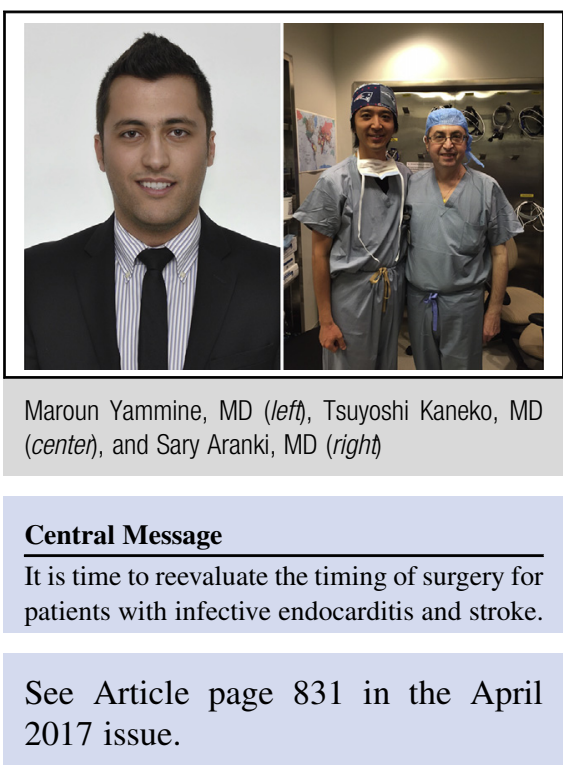

suspected or confirmed IE. Asymptomatic emboli may be a marker of a friable vegetation regardless of location, microorganism, or size. Early surgery may be indicated, with good outcomes such as those demonstrated in this study.

Of course, the weakness of this study relates to the fact that it is an uncontrolled, retrospective study, spanning a 24 -year period. Murai and coworkers ${ }^{1}$ went to great lengths in highlighting the limitations and weaknesses of this study, and they are to be commended for this care.

This is a well-thought-out and well-written study, one that we believe should be of interest to the multidisciplinary teams that manage patients in the acute phase of IE with cerebral complications. There is a grey zone as far as the timing of IE surgery is concerned. Current existing guidelines are either absent or at best fuzzy. Consequently, no two members of the various teams can agree on this issue. We hope that this study of Murai and coworkers, ${ }^{1}$ and others recently published, ${ }^{2,3}$ will allow us to think outside this nonexistent box and focus our decision making pertaining to the optimal timing of IE surgery.

\section{References}

1. Murai R, Funakoshi S, Kaji S, Kitai T, Kim K, Koyama T, et al. Outcomes of early surgery for infective endocarditis with moderate cerebral complications. J Thorac Cardiovasc Surg. 2017;153:831-40.e8.

2. Piper C, Wiemer M, Schulte HD, Horstkotte D. Stroke is not a contraindication for urgent valve replacement in acute infective endocarditis. J Heart Valve Dis. 2001; 10:703-11.

3. Yoshioke D, Sakaguchi T, Yamauchi T, Okazaki S, Miyagawa S, Nishi H, et al. Impact of early surgical treatment on postoperative neurologic outcome for active infective endocarditis complicated by cerebral infarction. Ann Thorac Surg. 2012; 94:489-95; discussion 496. 